\title{
Quiste hidatídico en ventrículo derecho
}

\author{
Enrique Seguel $^{1,2}$, Roberto González ${ }^{1,2}$, Aleck Stockins ${ }^{1,2}$, Felipe Caro. ${ }^{3}$ \\ 1. Hospital Guillermo Grant Benavente de Concepción, Centro Cardiovascular. \\ 2. Universidad de Concepción, Facultad de Medicina, Departamento de Cirugía. \\ 3. Universidad de Concepción, Alumno de Medicina.
}

\section{Right ventricular hydatid cyst}

A 20-year-old woman, without known pre-existent conditions presented with a history of dyspnea on exertion, and palpitations for 6 months. Vital sig$\mathrm{ns}$, as well as cardiac and pulmonary examinations were normal. Routine blood tests were normal. The ECG showed nonspecific ST-T changes. The echocardiogram showed a left ventricle of normal size and function. A cystic image was shown in relation to the right ventricle, with displacement of the interventricular septum. These findings were confirmed on computed tomography. Additional cystic images on the liver or lungs were ruled out. On surgery, a cardiac hydatic cyst adhered to the pericardium next to the right ventricle was found. Puncture, drainage of the mass and surgical removal of cystic membranes were performed. The clinical course was uneventful. A four year follow up revealed no recurrence of the cyst.

Keywords: Hydatid cyst, cardiac; echinococcosis, cardiac; surgery, cardiac. 


\section{Introducción:}

La enfermedad hidatídica o hidatidosis, es una parasitosis que se produce por la forma larvada de la tenia Echinococcus granulosus. Se ha descrito como una enfermedad de distribución mundial, con endemia en países latinoamericanos como Chile, Perú, Uruguay, Brasil y Argentina. La incidencia de la enfermedad hidatídica en nuestro país es de un 1.4/100.000 habitantes (año 2010) ${ }^{1}$.

La enfermedad hidatídica se ha caracterizado por su gran impacto en la salud pública, por ser una condición de diagnóstico tardío y con un alto costo en su tratamiento ${ }^{1,2}$.

La presentación clínica de la enfermedad hidatídica depende del órgano afectado y la concurrencia de complicaciones, existiendo un grupo importante de pacientes que adquieren la enfermedad y se mantienen asintomáticos. En estos casos la presencia del quiste resulta en un hallazgo imagenológico ${ }^{3}$.

Los quistes hidatídicos pueden existir en cualquier órgano. Sin embargo, sus localizaciones más frecuentes son el hígado ( $2 / 3$ de los casos) y el pulmón (1/5 de los casos $)^{4}$. Otras localizaciones menos frecuentes incluyen bazo, huesos, riñones, cerebro, corazón, peritoneo y tejido celular subcutáneo ${ }^{5}$. Hasta un $80 \%$ de los pacientes tienen compromiso de solo un órgano, existiendo en éste a su vez, un solo quiste. El compromiso cardíaco en la enfermedad hidatídica es poco frecuente y representa entre el $0,5 \%$ y $2 \%$ de los pacientes con hidatidosis 6 .

La afección cardíaca puede producirse por tres distintos mecanismos: la extensión desde estructuras comprometidas cercanas al corazón, la diseminación desde la circulación sistémica o la diseminación proveniente desde la circulación pulmonar ${ }^{7}$.

Se presenta el caso de una paciente portadora de esta patología, atendida en el Hospital Guillermo Grant Benavente de Concepción.

\section{Caso clínico:}

Una mujer de 20 años, sin antecedentes mórbidos conocidos, procedente de un sector rural cercano a Longaví en la Región del Maule, consultó por historia de disnea de esfuerzos y palpitaciones de 6 meses de evo-

Figura 1: Ecocardiograma trans torácico en que se observa imagen quística en el apex del corazón.

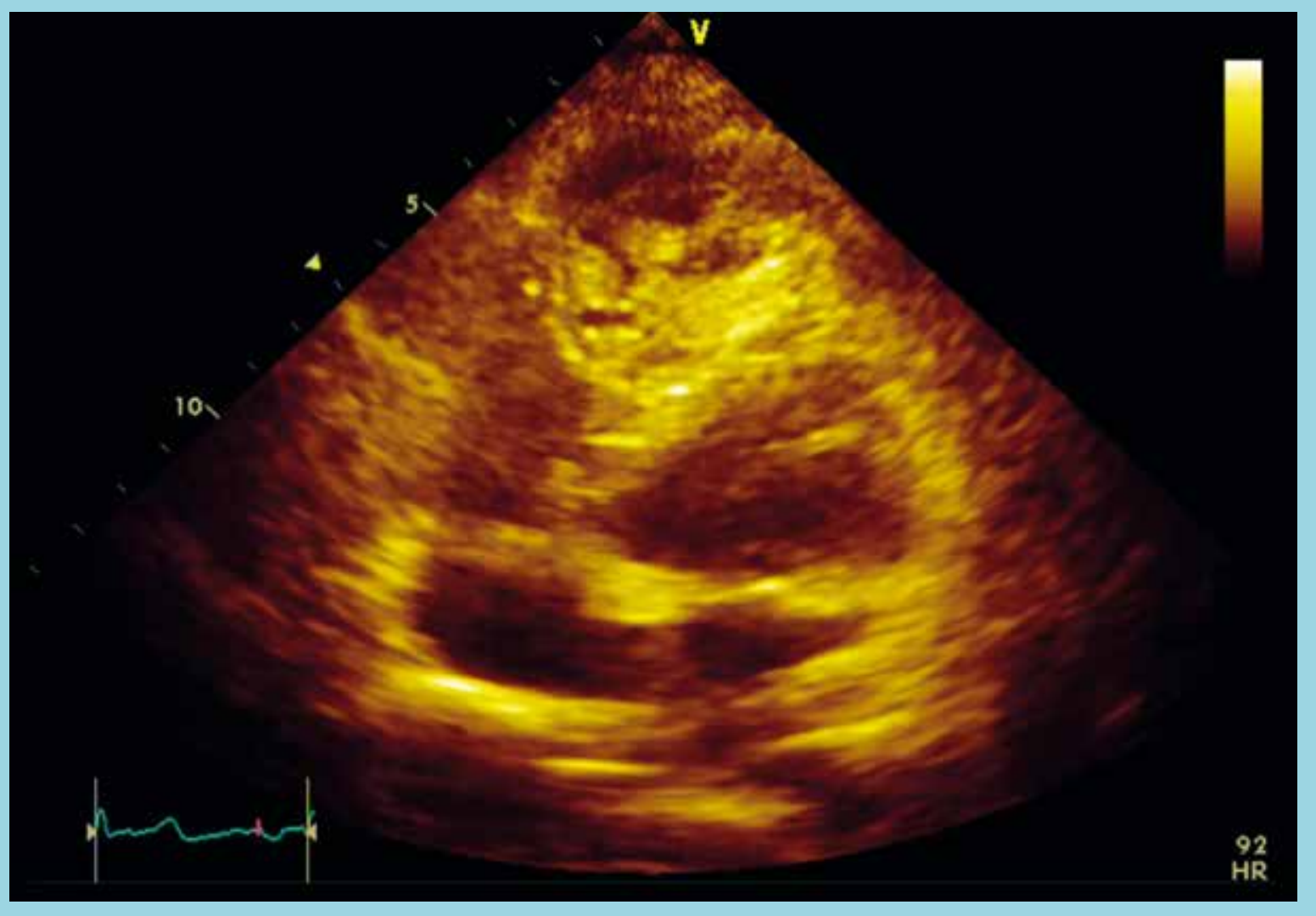




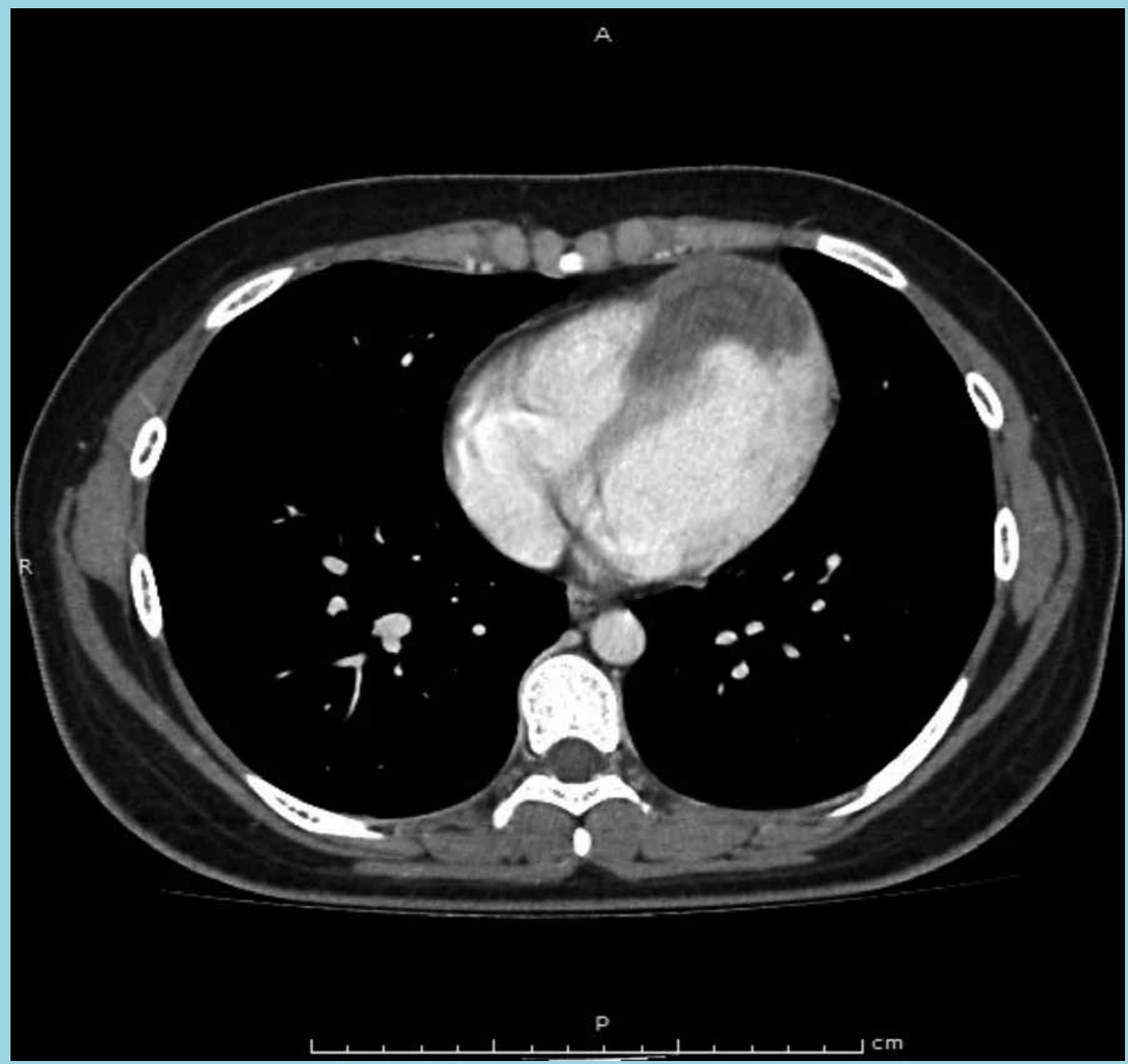

lución. Sus signos vitales y el examen cardíaco y pulmonar eran normales.

Los resultados de exámenes bioquímicos habituales fueron normales. El electrocardiograma evidenció alteraciones de la repolarización ventricular propias de sobrecarga de ventrículo izquierdo. Inicialmente, un ecocardiograma transtorácico mostró un ventrículo izquierdo de tamaño y función normales. Se identificó una imagen quística en relación con el ventrículo derecho y desplazamiento del tabique interventricular (Figura 1).

Una tomografía computarizada de tórax, abdomen y pelvis mostró una masa cardíaca, compatible con los hallazgos del ecocardiograma (Figura2). Además, se descartó la presencia concomitante de quistes en híga- do, pulmones u otros órganos. Se planteó la sospecha de un quiste hidatídico cardíaco y se decidió su tratamiento quirúrgico.

La cirugía se realizó bajo anestesia general y el corazón se abordó por esternotomía media. El corazón era de tamaño, morfología y contractilidad normales. A nivel de la punta del ventrículo derecho se observó una masa de aproximadamente $10 \mathrm{~cm}$ de diámetro, adherido al pericardio y con una zona necrótica en el centro (Figura 3). El corazón fue protegido con compresas y se aisló la zona mediante gasas con solución salina al $10 \%$. Se efectuó una punción y aspiración de la lesión, que dio salida a líquido, vesículas y membranas característicos de quiste hidatídico. Posteriormente, se destechó la cavidad y se extrajeron las membras y contenido. 
Figura 3: Imagen intra operatoria en la que se observa un aumento de volumen y una zona necrótica en el apex del corazón (flecha).

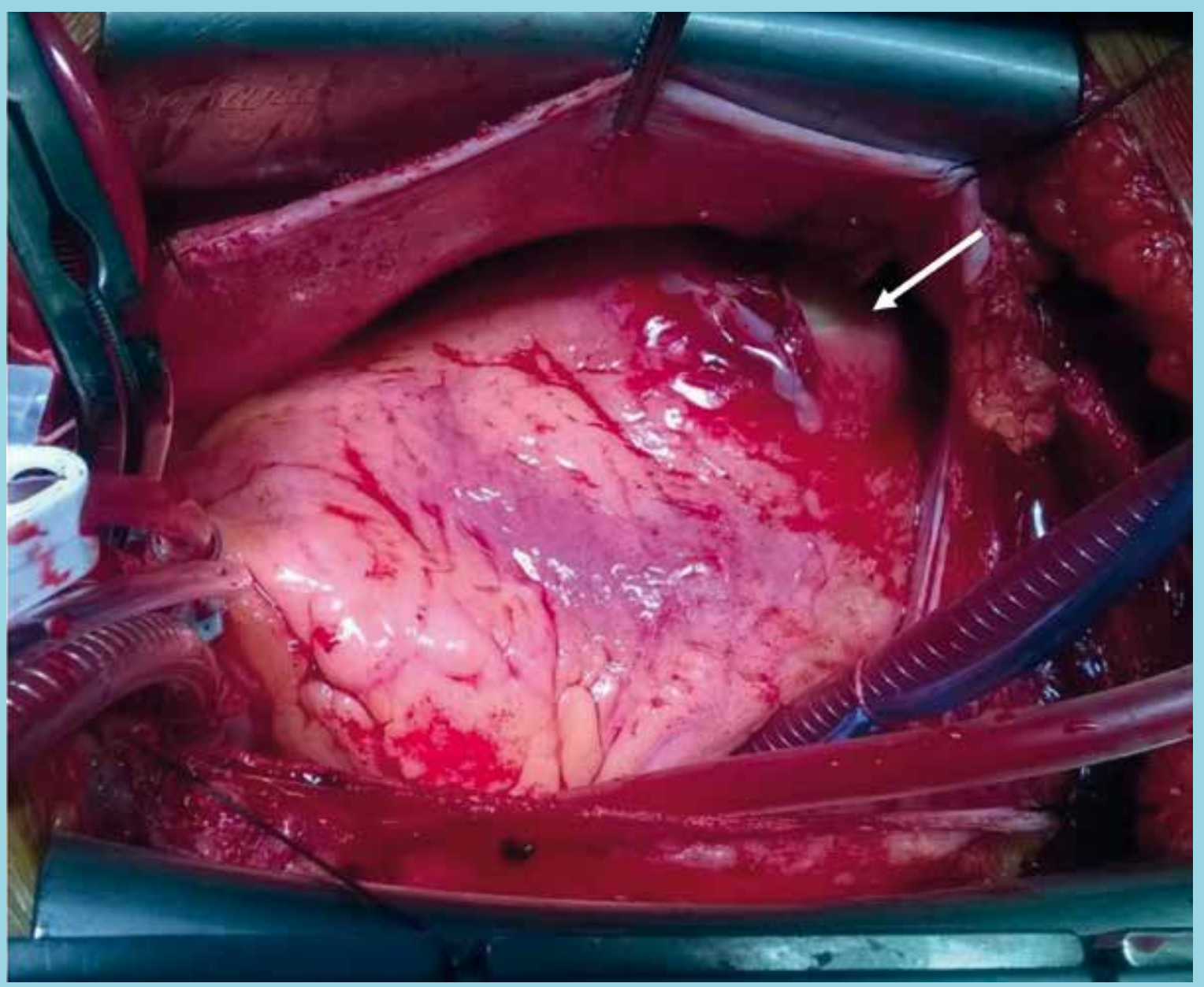

La cavidad peri quística comprometía septum y paredes ventriculares, pero sin comunicación con ellas. Se efectuó capitonaje de los bordes con sutura continua de polipropileno 5-0 y el cierre del defecto con sutura continua de polipropileno 3-0 en 2 planos.

El estudio anatomo-patológico confirmó que se trató de un quiste hidatídico (Figura 4).

Posterior a la cirugía, la paciente se recuperó sin complicaciones y fue dada de alta a su domicilio, con indicación de tratamiento con albendazol por tres meses.

En el seguimiento a cuatro años no existe evidencia de recidiva de la enfermedad.

\section{Discusión:}

La afección cardíaca por enfermedad hidatídica es una condición poco frecuente. No existen series grandes y en la literatura solo se pueden encontrar publicaciones con reportes de casos, especialmente en África, Medio
Oriente e India, con series de hasta 19 pacientes $^{8}$. La presentación clínica está determinada por la ubicación del quiste, su tamaño e integridad ${ }^{7}$. Distintas series muestran variabilidad respecto a la frecuencia en la localización de los quistes, predominando la ubicación en el ventrículo izquierdo (47\% - 75\%), ventrículo derecho $(15 \%-41,7 \%)^{7}-10$ y otras ubicaciones menos frecuentes como el tabique interventricular, pericardio, arteria pulmonar y aurícula derecha.

Existe una amplia gama de presentaciones clínicas, desde pacientes asintomáticos en quienes solo constituye un hallazgo ${ }^{10}$, hasta pacientes que presentan manifestaciones como disnea, arritmias, insuficiencia cardíaca $^{8,9}$, signos electrocardiográficos de isquemia, alteraciones en el segmento $\mathrm{ST}$ y onda $\mathrm{T}^{11}$, dolor precordial con electrocardiografía normal ${ }^{12}$, fiebre ${ }^{13,14}$ y síncope ${ }^{14,15}$.

El estudio diagnóstico, basado en el nivel de sospecha 
Figura 4: Membranas y quistes retirados del corazón.

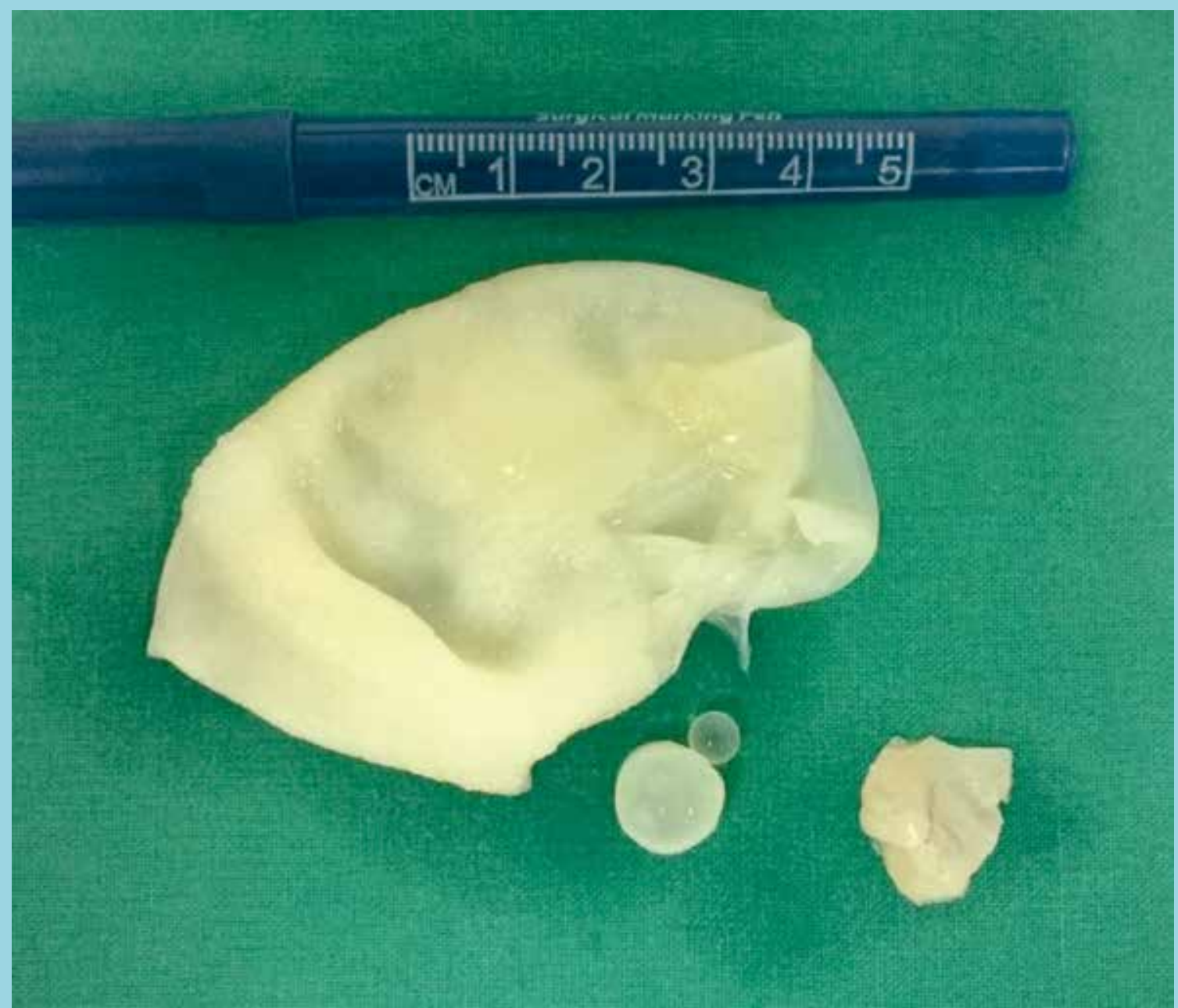

y la disponibilidad de recursos, puede incluir serología, electrocardiografía, estudios de imágenes como radiografía de tórax, tomografía computarizada, ecocardiograma transesofágico y transtorácico, angiografía coronaria y resonancia magnética $7-9,11-13,16$.

El tratamiento de elección es quirúrgico, describiéndose en la mayoría de los casos un abordaje vía esternotomía media, reservando otras vías de abordaje, como la toracotomía anterolateral, para locaciones de más difícil acceso ${ }^{10}$.

Como tratamiento complementario está descrito el uso de antiparasitarios como albendazol o mebendazol post cirugía, variando la dosis entre $400 \mathrm{mg}$ cada $12 \mathrm{~h}$ y 400 mg al día de albendazol. El uso del tratamiento médico con antiparasitarios está recomendado con el fin de reducir el riesgo de recurrencia, especialmente en aque- llos pacientes en que está comprometida la integridad del quiste ${ }^{11}$. Sin embargo, no hay acuerdo en cuanto a la prolongación del tratamiento que suele variar entre 6 meses y 5 años 15 .

El período de seguimiento está en estrecha relación con el tiempo total de tratamiento farmacológico post cirugía. Diversas series de casos reportan periodos de seguimiento que van desde los 6 meses hasta los 7 años. En los controles posteriores de la mayoría de las series no se describe recurrencia de la enfermedad, ni anormalidades cardíacas, existiendo sólo casos excepcionales de complicaciones que van desde la necesidad de reintervención a la muerte súbita ${ }^{7-11,16}$.

Entre los métodos de imagen utilizados en el seguimiento de los pacientes, se incluyen la resonancia magnética, la tomografía computarizada y el ecocardiograma. 


\section{Referencias}

1. INSTITUTO DE SALUD PÚBLICA DE CHILE. Vigilancia de Hidatidosis. Chile, 2010 - 2014. [Internet] Ispch.cl. 2015. [cited 29 July 2018]. Disponible en: http://www.ispch.cl/sites/default/files/Bolet\%C3\%ADn\%20Hidatidosis\%2030-032015.pdf.

2. MARTÍNEZ G P. Hidatidosis humana: antecedentes generales y situación epidemiológica en Chile, 2001-2009. Revista chilena de infectología. 2011; 28(6): 585 - 591.

3. PINTO GP. Diagnóstico, tratamiento y seguimiento de la hidatidosis. Revista Chilena de Cirugía. 2017;69 (1):94-98.

4. DA SILVAA. HUMAN ECHINOCOCCOSIS: A Neglected Disease. Gastroenterology Research and Practice. 2010;2010:1-9.

5. SALAMONE G. Uncommon localizations of hydatid cyst. Review of the literature. Giornale di Chirurgia - Journal of Surgery. 2016;

6. REIN R, NIGGEMANN B, RUNGE M. Echinococcosis of the heart. Herz. 1996 Jun;21 (3):192-7.

7. TEKIN A, DURMAZ M, DAĞLI M, AKBAYRAK S, AKBAYRAK P, TURGUT B. Left ventricular hydatid cyst mimicking acute coronary syndrome. Radiology Case Reports. 2018;13 (3):697-701.

8. NOAMAN H, RAWAF S, Majeed A, Salmasi A. Hydatid Cyst of the Heart. Angiology. 2017;68 (9):765-768.

9. FIENGO L, BUCCI F, GIANNOTTI D, PATRIZI G, REDLER A, KUCUKAKSU D. Giant Cardiac Hydatid Cyst in Children:
Case Report and Review of the Literature. Clinical Medicine Insights: Case Reports. 2014;7:CCRep.S15862.

10. TANYELI O, DERELI Y, MERCAN I, GORMUS N, YUKSEK T. New World's old disease: cardiac hydatid disease and surgical principles. Cardiovascular Journal of Africa. 2017;28 (5):304-308.

11. YALINIZ H, TOKCAN A, Salih OK, Ulus T. Surgical Treatment of Cardiac Hydatid Disease: A Report of 7 Cases. Texas Heart Institute Journal. 2006;33 (3):333-339.

12. GUNAY N, OCAL L, AKSOY S, BAKTIR A, KESER N. A Giant Isolated Cardiac Hydatid Cyst in the Interventricular Septum. Korean Circulation Journal. 2017;47(3):418.

13. FARAJI R, SABZI F, VAZIRI S. Right ventricular hydatid cyst ruptured to pericardium. Annals of Cardiac Anaesthesia. 2015;18(3):445.

14. MALIK V, DIVYA A, HOTE M, JAIN P. Interventricular septal hydatid cyst: Transesophageal echocardiography as a therapeutic tool during bypass. Annals of Cardiac Anaesthesia. 2015;18(3):421.

15. L'AARJE A, LYAZIDI S, KITANE Y, ALAMI A, HABBAL R. Cardiac hydatid cyst of the right ventricle: Severe localization. Journal of Cardiology Cases. 2017;16(4):138-140.

16. KAHLFUSS S, FLIEGER R, ROEPKE T, YILMAZ K. Diagnosis and treatment of cardiac echinococcosis. Heart. 2016;102(17):1348-1353. 